\title{
COUPLING OF TRANSPORT AND DIFFUSION MODELS IN LINEAR TRANSPORT THEORY
}

\author{
Guillaume BaL ${ }^{1}$ and YVON MadaY ${ }^{2}$
}

\begin{abstract}
This paper is concerned with the coupling of two models for the propagation of particles in scattering media. The first model is a linear transport equation of Boltzmann type posed in the phase space (position and velocity). It accurately describes the physics but is very expensive to solve. The second model is a diffusion equation posed in the physical space. It is only valid in areas of high scattering, weak absorption, and smooth physical coefficients, but its numerical solution is much cheaper than that of transport. We are interested in the case when the domain is diffusive everywhere except in some small areas, for instance non-scattering or oscillatory inclusions. We present a natural coupling of the two models that accounts for both the diffusive and non-diffusive regions. The interface separating the models is chosen so that the diffusive regime holds in its vicinity to avoid the calculation of boundary or interface layers. The coupled problem is analyzed theoretically and numerically. To simplify the presentation, the transport equation is written in the even parity form. Applications include, for instance, the treatment of clear or spatially inhomogeneous regions in near-infra-red spectroscopy, which is increasingly being used in medical imaging for monitoring certain properties of human tissues.
\end{abstract}

Mathematics Subject Classification. 45K05, 92C55, 65N55.

Received: August 5, 2001. Revised: November 6, 2001.

\section{INTRODUCTION}

Linear transport equations model the propagation of particles and of the energy density of high frequency waves in heterogeneous media. The particle description arises in physical phenomena such as the propagation of neutrons in nuclear reactor physics or electrons or photons in radiotherapy [17]. The wave description, leading to the theory of radiative transfer, models phenomena such as the propagation of electromagnetic waves in turbulent atmospheres and of shear waves generated by earthquakes in the earth's crust $[14,22,28,29]$.

The transport equations are posed in the phase space (position and velocity) and consequently quite expensive to solve numerically. They are therefore often replaced by a diffusion equation. Diffusion is valid provided scattering is large and absorption weak. In many physically interesting situations, the diffusion approximation is

\footnotetext{
Keywords and phrases. Linear transport, even parity formulation, diffusion approximation, domain decomposition, diffuse tomography.

${ }^{1}$ Department of Applied Physics \& Applied Mathematics, Columbia University, New York, NY 10027, USA.

e-mail: gb2030@columbia.edu

2 Laboratoire d'Analyse Numérique, Université Paris VI, boîte courrier 187, 75252 Paris Cedex 5, France.

e-mail: maday@ann.jussieu.fr
} 
valid in most of the domain, but not in some parts, where non-scattering inclusions are present, for instance. One way to deal with this issue is to generalize the diffusion model so that it also accounts for these inclusions $[4,8,10]$. A second more systematic way, the object of this paper, is to use the diffusion model where it is valid, use the transport model locally to treat the heterogeneities, and couple the two models using interface conditions at a judiciously chosen interface. A similar method based on the coupling of coarse and fine discretizations of transport has been studied in [7]. The coupling of transport and diffusion equations has also been considered in [30], with different coupling conditions than the ones in this paper, and in [23], where transport is solved by a Monte Carlo technique. Let us mention that the coupling of non-linear Boltzmann transport equations with their hydrodynamic limit (Euler equations) has also been addressed in the literature. We refer to [12,24,31] and their references.

The two main types of inclusions we consider are weakly diffusive inclusions and strongly absorbing inclusions, both being poorly captured by diffusion. An important application can be found in medical imaging through turbid media. Near-infra-red (NIR) spectroscopy, consisting of sending non-destructive NIR photons to probe a domain of interest, is increasingly being used for monitoring certain properties of human tissues [18,21]. The presence of clear and absorbing inclusions hampers the use of the diffusion model. Another application is the simulation of accidental depressurizations that can occur in nuclear reactors [5].

This paper deals with the steady state equations, although all results extend to the time dependent setting. The theory is based on the even parity formulation of transport [27]. This formulation is not as general as the classical first-order formulation of transport (see (1) below). However it admits a variational formulation that lends itself very naturally to the derivation of the diffusion approximation as an orthogonal projection for a suitable scalar product on the set of velocity-independent functions.

The paper is organized as follows. Section 1 recalls the derivation of the even parity formulation and presents the scaling that corresponds to the diffusion approximation. Section 2 is devoted to the derivation of the coupled problem, obtained by orthogonally projecting the transport solution on a set of functions that are velocity-independent only on some part of the domain. Convergence of the coupled problem and error estimates are given in Section 3. Section 4 deals with a relaxation iterative procedure, borrowed from the domain decomposition theory of elliptic equations, that allows us to numerically solve the coupled problem. Next, Section 5 presents the equivalent coupling between transport in its first-order formulation and diffusion. This is the coupled problem that is solved numerically. The numerical results are given in Section 6 , where the efficiency of the coupled problem is shown in cases of strongly absorbing and weakly scattering inclusions.

\section{EVEN PARITY FORMULATION AND DIFFUSION SCALING}

The phase space particle density $u(x, v)$ solves the following first-order linear transport equation

$$
\begin{aligned}
& v \cdot \nabla u(x, v)+\Sigma(x) u(x, v)=K u+q(x) \quad \text { in } \Omega \times V \\
& u(x, v)=g(x, v) \quad \text { on } \Gamma_{-}=\{(x, v) \in \partial \Omega \times V \text { s.t. } v \cdot \nu(x)<0\} .
\end{aligned}
$$

Here, $\Omega$ is the physical domain, a regular open bounded subdomain in $\mathbb{R}^{n}$ where $n=2,3$ is the space dimension. The velocity domain is denoted by $V$, a closed bounded subdomain in $\mathbb{R}^{n}$. Typically, $V$ is a finite union of spheres and/or coronas of various radii centered at $v=0$, with a measure $\mu$, the surface measure for spheres and the Lebesgue measure for coronas, normalized such that $\mu(V)=1$. In this paper, we assume that $V=S^{n-1}$, the unit sphere in $\mathbb{R}^{n}$. Furthermore, we denote by $\Sigma(x)=\sigma_{s}(x)+\sigma_{a}(x)$ the total absorption coefficient. It is the sum of the intrinsic absorption coefficient $\sigma_{a}(x)$ and the scattering coefficient $\sigma_{s}(x)$. The scattering operator is defined by

$$
K u(x, v)=\sigma_{s}(x) \int_{V} f\left(v^{\prime}, v\right) u\left(x, v^{\prime}\right) \mathrm{d} \mu\left(v^{\prime}\right), \quad \text { for all }(x, v) \in \Omega \times V
$$


where $f\left(v^{\prime}, v\right)$ is a bounded positive function satisfying

$$
\int_{V} f\left(v, v^{\prime}\right) \mathrm{d} \mu\left(v^{\prime}\right)=1
$$

We only consider the isotropic case $f \equiv 1$ to simplify the presentation.

The leading differential operator $v \cdot \nabla$ is not symmetric. This causes some difficulties in the theoretical analysis of the transport equation [16]. One way to symmetrize the transport problem is to have recourse to its even parity formulation [27]. Let us introduce the even and odd fluxes

$$
\psi(x, v)=\frac{1}{2}(u(x, v)+u(x,-v)), \quad \psi^{-}(x, v)=\frac{1}{2}(u(x, v)-u(x,-v))
$$

Subtracting (1) at $-v$ from (1) at $v$ yields

$$
\psi^{-}(x, v)=-\frac{1}{\Sigma(x)} v \cdot \nabla \psi(x, v) .
$$

Upon adding these same equations and replacing $\psi^{-}(x, v)$ by its expression in $(4)$, we obtain

$$
\begin{aligned}
& -v \cdot \nabla \frac{1}{\Sigma} v \cdot \nabla \psi(x, v)+\Sigma(x) \psi(x, v)=K \psi+q(x) \quad \text { in } \quad \Omega \times V, \\
& \psi-\frac{1}{\Sigma} v \cdot \nabla \psi=g(x, v) \quad \text { on } \quad \Gamma_{-} .
\end{aligned}
$$

This procedure relies on the specific nature of the velocity space $V$, which must be symmetric, and of the scattering operator $K$, which must be isotropic. The even parity formulation can be derived for more complicated settings, but it is less general that the first-order transport equation [27].

The main feature of this formulation is that its leading differential term $-v \cdot \nabla \frac{1}{\Sigma} v \cdot \nabla$ is now symmetric. Indeed, upon multiplying (5) by the test function $\theta$ and integrating by parts, we recast (5) as

$$
a(\psi, \theta)=(q, \theta)+\langle g, \theta\rangle, \quad \forall \theta \in \mathcal{V}
$$

Here $(\cdot, \cdot)$ is the standard $L^{2}(\Omega \times V)$ product and

$$
\langle f, g\rangle=\int_{\partial \Omega} \int_{V} f(x, v) g(x, v)|v \cdot \nu(x)| \mathrm{d} \sigma(x) \mathrm{d} \mu(v),
$$

where $d \sigma(x)$ is the surface measure on $\partial \Omega$. The bilinear form $a(\psi, \theta)$ is given by

$$
\begin{aligned}
a(\psi, \theta)= & \int_{\Omega} \int_{V}\left(\frac{1}{\Sigma} v \cdot \nabla \psi v \cdot \nabla \theta+\sigma_{a} \psi \theta\right) \mathrm{d} x \mathrm{~d} \mu(v) \\
& +\int_{\partial \Omega} \int_{V}|v \cdot \nu(x)| \psi \theta \mathrm{d} d \sigma(x) \mathrm{d} \mu(v) \\
& +\int_{\Omega} \int_{V} \sigma_{s}(x)\left(\psi(x, v)-\int_{V} \psi\left(x, v^{\prime}\right) d \mu\left(v^{\prime}\right)\right) \theta(x, v) \mathrm{d} x \mathrm{~d} \mu(v) .
\end{aligned}
$$

This is deduced from the relation $\psi(x,-v)=\psi(x, v)$. The two first terms in the definition of the bilinear form $a$ are clearly symmetric. It is not difficult to convince oneself that the third term also is symmetric. We therefore have a symmetric bilinear form $a$, and its domain of definition is the Hilbert space $\mathcal{V}$ defined by

$$
\mathcal{V}=\left\{\psi \in L^{2}(\Omega \times V) \text { s.t. } v \cdot \nabla \psi \in L^{2}(\Omega \times V) \text { and }|v \cdot \nu|^{\frac{1}{2}} \psi \in L^{2}(\partial \Omega \times V)\right\}
$$


In this definition, it is also implicitly understood that $\psi(x,-v)=\psi(x, v)$. We define $L_{|v \cdot \nu|}^{2}(\partial \Omega \times V)$ as the space of functions $\psi$ such that $|v \cdot \nu|^{\frac{1}{2}} \psi \in L^{2}(\partial \Omega \times V)$. The space $\mathcal{V}$ is equipped with its natural norm $\|\cdot\|_{\mathcal{V}}$.

Assuming that the scattering coefficient $\sigma_{s}$ is uniformly bounded above and below by positive constants and that the absorption coefficient $\sigma_{a}$ is non-negative and bounded, we can show that the bilinear form $a$ is continuous and coercive for the natural norm in $\mathcal{V}$ (this is easily obtained when $\sigma_{a}$ is uniformly positive, and requires a Poincaré-like inequality [3] when $\sigma_{a}$ is non-negative). We deduce from the Lax-Milgram theorem the existence of a unique solution to (6) in $\mathcal{V}$.

The diffusion approximation holds when the medium is highly scattering and weakly absorbing. We introduce the small parameter $\varepsilon$, which measures the mean free path, the characteristic distance between two successive interactions of a particle with the underlying medium. High scattering is modeled by replacing $\sigma_{s}$ by $\sigma_{s \varepsilon} / \varepsilon$ and weak absorption by replacing $\sigma_{a}$ by $\varepsilon \sigma_{a \varepsilon}$. The coefficients $\sigma_{s \varepsilon}$ and $\sigma_{a \varepsilon}$ are allowed to depend on $\varepsilon$, but we impose that they converge strongly in the $L^{\infty}$ sense. The source term $q(x)$ is also replaced by $\varepsilon q_{\varepsilon}(x)$ to obtain solutions of the transport equation that are bounded independent of $\varepsilon$. Upon replacing $\sigma_{s}$ and $\sigma_{a}$ by their scaled versions in (7) and dividing by $\varepsilon$, we define the scaled bilinear form

$$
\begin{aligned}
a_{\varepsilon}(\psi, \theta)= & \int_{\Omega} \int_{V}\left(\frac{1}{\sigma_{s \varepsilon}+\varepsilon^{2} \sigma_{a \varepsilon}} v \cdot \nabla \psi v \cdot \nabla \theta+\sigma_{a \varepsilon} \psi \theta\right) \mathrm{d} x \mathrm{~d} \mu(v) \\
& +\frac{1}{\varepsilon} \int_{\partial \Omega} \int_{V}|v \cdot \nu(x)| \psi \theta \mathrm{d} \sigma(x) \mathrm{d} \mu(v) \\
& +\int_{\Omega} \int_{V} \frac{\sigma_{s \varepsilon}(x)}{\varepsilon^{2}}(\psi(x, v)-\bar{\psi}(x)) \theta(x, v) \mathrm{d} x \mathrm{~d} \mu(v) .
\end{aligned}
$$

Here, $\bar{\psi}(x)=\int_{V} \psi(x, v) \mathrm{d} \mu(v)$. This is the classical diffusion scaling. The derivation of boundary conditions for diffusion from inhomogeneous transport boundary conditions is involved and treated in the literature $[11,16]$. To concentrate on the transport-diffusion coupling, we assume in the coming sections that we have vanishing incoming boundary conditions: $g=0$. The rescaled transport problem (6) then reads

$$
a_{\varepsilon}\left(\psi_{\varepsilon}, \theta\right)=\left(q_{\varepsilon}, \theta\right) \quad \forall \theta \in \mathcal{V} .
$$

Provided that $q_{\varepsilon}$ remains uniformly bounded in $L^{2}(\Omega \times V)$, the transport problem (13) admits a unique and uniformly bounded solution in $\mathcal{V}$.

\section{Coupled PROBlem}

The diffusion regime is characterized by the asymptotic expansion

$$
\psi_{\varepsilon}(x, v)=\bar{\psi}_{\varepsilon}(x)+O\left(\varepsilon^{2}\right),
$$

at least far away from boundaries. The physical reason is that particles scatter often with the underlying medium before being absorbed or leaving the domain. Consequently, they lose their initial directional information and quickly relax to the local equilibrium, which here means to be velocity independent. That the error is of order $O\left(\varepsilon^{2}\right)$ can be read off from asymptotic expansions $[6,16]$ (see also Th. 3.2 below).

We split the physical domain $\Omega$ into two subdomains $\Omega_{\mathrm{di}}$ and $\Omega_{\mathrm{tr}}$. Their common boundary $\gamma$ is supposed to be smooth, with possibly several connected components. We assume the diffusion regime to hold in $\Omega_{\mathrm{di}}$ but not necessarily in $\Omega_{\mathrm{tr}}$. This forces $\psi$ to be independent of $v$ in $\Omega_{\mathrm{di}}$, but not in $\Omega_{\mathrm{tr}}$. On the other hand, the bilinear form $a_{\varepsilon}$ is symmetric in $\mathcal{V}$. A natural choice for a coupled solution to be velocity independent in $\Omega_{\mathrm{di}}$ but not in $\Omega_{\mathrm{tr}}$, is to project the solution $\psi_{\varepsilon}$ on the subset $\hat{\mathcal{V}}$ of $\mathcal{V}$ of functions independent of $v$ on $\Omega_{\mathrm{di}}$, parallel to the orthogonal subset $\mathcal{W}_{\varepsilon}$ of $\mathcal{V}$ for the bilinear form $a_{\varepsilon}$. More specifically, we define

$$
\hat{\mathcal{V}}=\left\{\theta \in \mathcal{V} \quad \text { s.t. } \quad \theta=\theta(x) \quad \text { on } \quad \Omega_{\mathrm{di}}\right\} .
$$


We check that $\hat{\mathcal{V}}$ is a closed subspace of $\mathcal{V}$ and define

$$
\mathcal{W}_{\varepsilon}=\left\{w \in \mathcal{V} \quad \text { s.t. } \quad a_{\varepsilon}(w, \theta)=0 \quad \forall \theta \in \hat{\mathcal{V}}\right\}
$$

Since $a_{\varepsilon}$ is coercive on $\mathcal{V}$, we have $\hat{\mathcal{V}} \cap \mathcal{W}_{\varepsilon}=\{0\}=\hat{\mathcal{V}}^{\perp} \cap \mathcal{W}_{\varepsilon}^{\perp}$. Hence, $\mathcal{V}=\hat{\mathcal{V}} \oplus \mathcal{W}_{\varepsilon}$.

Let us introduce the orthogonal projection $\Pi_{\varepsilon}$ of $\mathcal{V}$ on $\hat{\mathcal{V}}$ for the scalar product $a_{\varepsilon}(\cdot, \cdot)$. We define the solution of the coupled problem $\psi_{\varepsilon}^{c}$ as

$$
\psi_{\varepsilon}^{c}=\Pi_{\varepsilon} \psi_{\varepsilon}
$$

where $\psi_{\varepsilon}$ is the solution to the transport problem (13). By definition, $\psi_{\varepsilon}^{c}$ solves the following problem

$$
a_{\varepsilon}\left(\psi_{\varepsilon}^{c}, \theta\right)=\left(q_{\varepsilon}, \theta\right) \quad \forall \theta \in \hat{\mathcal{V}}
$$

This problem does not quite look like a coupled transport-diffusion problem yet. Let us introduce

$$
\begin{aligned}
& \psi_{\mathrm{tr}}^{c}=\psi_{\varepsilon}^{c} \quad \text { on } \quad \Omega_{\mathrm{tr}} \times V, \\
& \psi_{\mathrm{di}}^{c}=\psi_{\varepsilon}^{c} \text { on } \Omega_{\mathrm{di}},
\end{aligned}
$$

and let $\theta \in \mathcal{D}\left(\Omega_{\mathrm{di}}\right) \subset \hat{\mathcal{V}}$ be a test function. We obtain that

$$
\int_{\Omega_{\mathrm{di}}} \int_{V}\left(\frac{1}{\Sigma_{\varepsilon}} v \cdot \nabla \psi_{\mathrm{di}}^{c} v \cdot \nabla \theta+\sigma_{a \varepsilon} \psi_{\mathrm{di}}^{c} \theta\right) \mathrm{d} \mu(v) \mathrm{d} x=\left(q_{\varepsilon}, \theta\right) .
$$

Here, $\Sigma_{\varepsilon}=\sigma_{s \varepsilon}+\varepsilon^{2} \sigma_{a \varepsilon}$. Since neither $\theta$ nor $\psi_{\mathrm{di}}^{c}$ depend on $v$, and since $\int_{V} v_{i} v_{j} \mathrm{~d} \mu(v)=n^{-1} \delta_{i j}$ (where $n=2,3$ is the space dimension), we obtain after integrations by parts that

$$
-\operatorname{div} D_{\varepsilon} \nabla \psi_{\mathrm{di}}^{c}+\sigma_{a \varepsilon} \psi_{\mathrm{di}}^{c}=q_{\varepsilon} \quad \text { in } \quad \Omega_{\mathrm{di}}
$$

in the weak sense. The diffusion coefficient is given by

$$
D_{\varepsilon}(x)=\frac{1}{n \Sigma_{\varepsilon}(x)}
$$

It depends on $\varepsilon$, and behaves as $\left(n \sigma_{s \varepsilon}\right)^{-1}$ when $\varepsilon \rightarrow 0$.

Similarly, choosing now $\theta \in \mathcal{D}\left(\Omega_{\mathrm{tr}} \times V\right)$ (see [16] for a definition of this space), we obtain that

$$
-v \cdot \nabla \frac{\varepsilon}{\Sigma_{\varepsilon}} v \cdot \nabla \psi_{\mathrm{tr}}^{c}+\varepsilon \sigma_{a \varepsilon} \psi_{\mathrm{tr}}^{c}+\frac{1}{\varepsilon} \sigma_{s \varepsilon}\left(\psi_{\mathrm{tr}}^{c}-\bar{\psi}_{\mathrm{tr}}^{c}\right)=\varepsilon q_{\varepsilon} \quad \text { in } \Omega_{\mathrm{tr}} \times V .
$$

It remain to address the boundary conditions. We distinguish three parts of the boundary: $\partial \Omega \cap \partial \Omega_{\mathrm{di}}, \partial \Omega \cap \partial \Omega_{\mathrm{tr}}$, and $\gamma$, provided these sets are not empty. We then multiply (20) and (22) by test functions $\theta \in \hat{\mathcal{V}}$ that do not vanish on only one of the three boundaries.

Let us first consider $\partial \Omega \cap \partial \Omega_{\mathrm{di}}$ (assuming it is not empty). We remark that

$$
c_{n}=\int_{V}|v \cdot \nu(x)|=\frac{1}{2} \quad \text { for } \quad n=3 \quad \text { and } \quad c_{n}=\frac{2}{\pi} \quad \text { for } \quad n=2
$$

For every test function $\theta$ vanishing on $\partial \Omega \cap \partial \Omega_{\operatorname{tr}}$ and $\gamma$, we have

$$
-\int_{\partial \Omega \cap \partial \Omega_{\mathrm{di}}} D_{\varepsilon} \frac{\partial \psi_{\mathrm{di}}^{c}}{\partial \nu} \theta \mathrm{d} \sigma=\int_{\partial \Omega \cap \partial \Omega_{\mathrm{di}}} \frac{1}{\varepsilon} c_{n} \psi_{\mathrm{di}}^{c} \theta \mathrm{d} \sigma .
$$


This yields the classical boundary condition

$$
c_{n} \psi_{\mathrm{di}}^{c}+\varepsilon D_{\varepsilon} \frac{\partial \psi_{\mathrm{di}}^{c}}{\partial \nu}=0 \quad \text { on } \quad \partial \Omega \cap \partial \Omega_{\mathrm{di}}
$$

We now turn our attention to $\partial \Omega \cap \partial \Omega_{\mathrm{tr}}$ (again assuming it is not empty). Following the same procedure, we get the following boundary conditions for the even form of the transport equation

$$
\psi_{\mathrm{tr}}^{c}-\frac{\varepsilon}{\Sigma_{\varepsilon}} v \cdot \nabla \psi_{\mathrm{tr}}^{c}=0 \quad \text { on } \Gamma_{-} \cap\left(\partial \Omega_{\mathrm{tr}} \times V\right) .
$$

Since $\psi_{\operatorname{tr}}^{c}(x,-v)=\psi_{\operatorname{tr}}^{c}(x, v)$, the condition is defined on the whole phase space boundary $\left(\partial \Omega \cap \partial \Omega_{\mathrm{tr}}\right) \times V$.

It remains to address the interface conditions on $\gamma$. Proceeding as above, we obtain that

$$
\frac{1}{\Sigma} \int_{V} v \cdot \nu_{\gamma}(x) v \cdot \nabla \psi_{\mathrm{tr}}^{c}=D_{\varepsilon} \frac{\partial \psi_{\mathrm{di}}^{c}}{\partial \nu_{\gamma}} \quad \text { on } \quad \gamma
$$

where $\nu_{\gamma}(x)$ is the outward unit normal vector to $\Omega_{\mathrm{tr}}$ at $x \in \gamma$. Since $\psi^{c} \in \hat{\mathcal{V}}$, we also deduce that $\psi_{\mathrm{tr}}^{c}=\psi_{\mathrm{di}}^{c}$ on $\gamma$. Hence, as for every second-order differential equation, two interface conditions are imposed at the interface $\gamma$. The above calculations are summarized in the following coupled transport-diffusion problem: Find $\psi_{\varepsilon}^{c}=\left(\psi_{\mathrm{tr}}^{c}, \psi_{\mathrm{di}}^{c}\right) \in \hat{\mathcal{V}}$ solving

$$
\begin{aligned}
& -\operatorname{div} D_{\varepsilon} \nabla \psi_{\mathrm{di}}^{c}+\sigma_{a \varepsilon} \psi_{\mathrm{di}}^{c}=q_{\varepsilon} \quad \text { in } \quad \Omega_{\mathrm{di}}, \\
& c_{n} \psi_{\mathrm{di}}^{c}+\varepsilon D_{\varepsilon} \frac{\partial \psi_{\mathrm{di}}}{\partial \nu}=0 \quad \text { on } \quad \partial \Omega \cap \partial \Omega_{\mathrm{di}}, \\
& -v \cdot \nabla \frac{1}{\sum_{\varepsilon}} v \cdot \nabla \psi_{\mathrm{tr}}^{c}+\sigma_{a \varepsilon} \psi_{\mathrm{tr}}^{c}+\frac{1}{\varepsilon^{2}} \sigma_{s \varepsilon}\left(\psi_{\mathrm{tr}}^{c}-\bar{\psi}_{\mathrm{tr}}^{c}\right)=q_{\varepsilon} \quad \text { in } \Omega_{\mathrm{tr}} \times V, \\
& \psi_{\mathrm{tr}}^{c}-\frac{{ }^{2}}{\Sigma_{\varepsilon}} v \cdot \nabla \psi_{\mathrm{tr}}^{c}=0 \quad \text { on } \quad \Gamma_{-} \cap\left(\partial \Omega_{\mathrm{tr}} \times V\right), \\
& \psi_{\mathrm{tr}}^{c}=\psi_{\mathrm{di}}^{c} \quad \text { on } \quad \gamma \times V, \\
& \frac{1}{\Sigma} \int_{V} v \cdot \nu_{\gamma}(x) v \cdot \nabla \psi_{\mathrm{tr}}^{c}=D_{\varepsilon} \frac{\partial \psi_{\mathrm{di}}^{c}}{\partial \nu_{\gamma}} \quad \text { on } \quad \gamma .
\end{aligned}
$$

We have shown that the above coupled problem is equivalent to (18) and thus admits a unique (weak) solution in $\hat{\mathcal{V}}$.

\section{Convergence And ERror estimates}

This section shows that the difference between the solution of the coupled problem (27) and that of the transport problem (13) tends to 0 as $\varepsilon \rightarrow 0$. The main result is as follows

Theorem 3.1. Assume that the coefficients $\sigma_{s \varepsilon}$ and $\sigma_{a \varepsilon}$ are uniformly bounded above and below by positive constants and that they converge strongly to $\sigma_{s}$ and $\sigma_{a}$, respectively, in $L^{\infty}(\Omega)$. that

Then the difference between the solution $\psi_{\varepsilon}^{c}$ to (18) and the solution $\psi_{\varepsilon}$ to (13) converges to 0 , in the sense

$$
a_{\varepsilon}\left(\psi_{\varepsilon}-\psi_{\varepsilon}^{c}, \psi_{\varepsilon}-\psi_{\varepsilon}^{c}\right) \rightarrow 0 \quad \text { as } \quad \varepsilon \rightarrow 0 .
$$

Since the bilinear form $a_{\varepsilon}$ is uniformly coercive, this also implies that

$$
\left\|\psi_{\varepsilon}-\psi_{\varepsilon}^{c}\right\|_{\mathcal{V}} \rightarrow 0 \quad \text { as } \quad \varepsilon \rightarrow 0
$$


Proof. Let us write $\psi_{\varepsilon}=\psi_{\varepsilon}^{c}+\delta \psi_{\varepsilon}$. By definition of the projection operator $\Pi_{\varepsilon}$, we have $\delta \psi_{\varepsilon} \in \mathcal{W}_{\varepsilon}$. For any test function $\theta \in \mathcal{V}$, we also write $\theta=\bar{\theta}+\tilde{\theta}$ with $\bar{\theta} \in \hat{\mathcal{V}}$ and $\tilde{\theta} \in \mathcal{W}_{\varepsilon}$. Such a decomposition is unique. Since $a_{\varepsilon}\left(\psi_{\varepsilon}, \tilde{\theta}\right)=a_{\varepsilon}\left(\delta \psi_{\varepsilon}, \tilde{\theta}\right)$, we deduce that

$$
a_{\varepsilon}\left(\delta \psi_{\varepsilon}, \tilde{\theta}\right)=\left(q_{\varepsilon}, \tilde{\theta}\right) \quad \forall \tilde{\theta} \in \mathcal{W}_{\varepsilon}
$$

Upon choosing $\tilde{\theta}=\delta \psi_{\varepsilon}$, we obtain that $\delta \psi_{\varepsilon}$ is bounded in $\mathcal{V}$ independent of $\varepsilon$. Since $\mathcal{V}$ is a Hilbert space, the unit ball in $\mathcal{V}$ is compact for the weak topology and $\delta \psi_{\varepsilon}$ converges, up to the extraction of subsequences, weakly to the function $w \in \mathcal{V}$. Owing to the form of $a_{\varepsilon}$ in (10), the integral $\varepsilon^{-1} \int_{\partial \Omega} \int_{V}|v \cdot \nu|\left(\delta \psi_{\varepsilon}\right)^{2}$ is uniformly bounded. Hence, in the limit $\varepsilon \rightarrow 0$, we have $w=0$ on $\partial \Omega \times V$.

That $\delta \psi_{\varepsilon}$ is bounded in $\mathcal{V}$ is not sufficient to obtain strong convergence of $\delta \psi_{\varepsilon}$ to $w$ in $L^{2}(\Omega \times V)$. However, it is sufficient to obtain that its velocity average $\overline{\delta \psi_{\varepsilon}}$ converges strongly to $\bar{w}$ in $L^{2}(\Omega)$. Here, we use an averaging lemma [20]. Also from (10), we deduce that

$$
\left\|\delta \psi_{\varepsilon}-\overline{\delta \psi_{\varepsilon}}\right\|_{L^{2}(\Omega \times V)} \leq C \varepsilon,
$$

hence tends to 0 as $\varepsilon \rightarrow 0$. Therefore, $\delta \psi_{\varepsilon}$, like its velocity average, converges to $\bar{w}$. This implies that $w=\bar{w}$ on $\Omega$, and thus $w \in \hat{\mathcal{V}}$. As a consequence, we have

$$
a_{\varepsilon}\left(\delta \psi_{\varepsilon}, w\right)=0 .
$$

The latter is equivalent to

$$
\int_{\Omega} \int_{V}\left(\frac{1}{\Sigma_{\varepsilon}} v \cdot \nabla \delta \psi_{\varepsilon} v \cdot \nabla w+\sigma_{a \varepsilon} \delta \psi_{\varepsilon} w\right) \mathrm{d} x \mathrm{~d} \mu(v)=0
$$

Since $\sigma_{a \varepsilon}$ and $\Sigma_{\varepsilon}$ converge strongly to $\sigma_{a}$ and $\sigma_{s}$ in $L^{\infty}(\Omega)$, we now let $\varepsilon \rightarrow 0$ in the above expression and get that

$$
\int_{\Omega}\left(\frac{1}{3 \sigma_{s}}(\nabla w)^{2}+\sigma_{a} w^{2}\right) \mathrm{d} x=0
$$

hence $w=0$ on $\Omega$ by the Poincaré inequality since $w=0$ on $\partial \Omega$.

The above result of convergence is solely based on the convergence properties of the coefficients $\sigma_{a \varepsilon}$ and $\sigma_{s \varepsilon}$ and does not rely on any regularity of the coupled solution $\psi_{\varepsilon}^{c}$ or of the exact solution $\psi_{\varepsilon}$. A similar purely variational approach was used to deal with the homogenization of highly oscillatory coefficients [3]. Error estimates are more difficult to obtain with purely variational techniques, and usually rely on the regularity of the macroscopic solution (here the coupled solution $\psi_{\varepsilon}^{c}$ ). We now present such an error estimate.

Let us define the distribution $\psi_{2}^{c}$ as the unique solution to

$$
\begin{aligned}
& \left(\sigma_{s \varepsilon} \psi_{2}^{c}, \theta\right)=\left(q_{\varepsilon}, \theta\right)-a_{\varepsilon}\left(\psi_{\varepsilon}^{c}, \theta\right) \quad \forall \theta \in \mathcal{V}, \\
& \int_{V} \psi_{2}^{c} \mathrm{~d} \mu(v)=0 .
\end{aligned}
$$

By integrations by parts, we check that $\psi_{2}^{c}$ is given by

$$
\sigma_{s \varepsilon} \psi_{2}^{c}=\left\{\begin{array}{lll}
0 & \text { on } & \Omega_{\mathrm{tr}} \times V, \\
v \cdot \nabla \frac{1}{\Sigma_{\varepsilon}} v \cdot \nabla \psi_{\varepsilon}^{c}-\operatorname{div} D_{\varepsilon} \nabla \psi_{\varepsilon}^{c} & \text { on } & \Omega_{\mathrm{di}} \times V .
\end{array}\right.
$$


Notice therefore that the support of $\psi_{2}^{c}$ is a subset of $\Omega_{\mathrm{di}} \times V$. Consider the following Ansatz

$$
\psi_{\varepsilon}=\psi_{\varepsilon}^{c}+\varepsilon^{2} \psi_{2}^{c}+\theta_{\varepsilon}
$$

Since $a_{\varepsilon}\left(\psi_{\varepsilon}, \theta\right)=\left(q_{\varepsilon}, \theta\right)$, we obtain that

$$
a_{\varepsilon}\left(\psi_{\varepsilon}^{c}, \theta\right)+\varepsilon^{2} a_{\varepsilon}\left(\psi_{2}^{c}, \theta\right)+a_{\varepsilon}\left(\theta_{\varepsilon}, \theta\right)=\left(q_{\varepsilon}, \theta\right) \quad \forall \theta \in \mathcal{V},
$$

or equivalently

$$
a_{\varepsilon}\left(\theta_{\varepsilon}, \theta\right)=-\varepsilon^{2}\left(a_{\varepsilon}\left(\psi_{2}^{c}, \theta\right)-\frac{1}{\varepsilon^{2}}\left(\sigma_{s \varepsilon} \psi_{2}^{c}, \theta\right)\right) .
$$

Choosing now $\theta=\theta_{\varepsilon}$, we deduce from (10), (31), and the Cauchy-Schwarz inequality that

$$
a_{\varepsilon}^{1 / 2}\left(\theta_{\varepsilon}, \theta_{\varepsilon}\right) \leq C \varepsilon^{2}\left(\left\|\psi_{2}^{c}\right\|_{L^{2}\left(\Omega_{\mathrm{di}} \times V\right)}+\left\|v \cdot \nabla \psi_{2}^{c}\right\|_{L^{2}\left(\Omega_{\mathrm{di}} \times V\right)}+\frac{1}{\varepsilon}\left\||v \cdot \nu|^{1 / 2} \psi_{2}^{c}\right\|_{L^{2}\left(\left(\partial \Omega \cap \partial \Omega_{\mathrm{di}}\right) \times V\right)}\right),
$$

since $\overline{\psi_{2}^{c}}=0$ and $\operatorname{supp}\left(\psi_{2}^{c}\right) \subset \Omega_{\mathrm{di}} \times V$. The same estimate clearly holds for $\varepsilon^{2} \psi_{\varepsilon}^{c}$ itself, hence for $\delta \psi_{\varepsilon}=\psi_{\varepsilon}-\psi_{\varepsilon}^{c}$.

We see here the effects of the transport-diffusion coupling. When $\partial \Omega \cap \partial \Omega_{\mathrm{di}}=\emptyset$, i.e., when the external boundary $\partial \Omega$ is treated in transport, the error is of order $\varepsilon^{2}\left\|\psi_{2}^{c}\right\|_{\mathcal{V}}$. When the external boundary is partially or totally treated with the diffusion approximation, the error is only of order $\varepsilon\left\|\psi_{2}^{c}\right\|_{\mathcal{V}}$, which is optimal. Indeed, the transport solution is not smooth in the vicinity of the boundary. Its description requires a boundary layer term, which is of order $\varepsilon[16]$ and cannot be accounted for by the diffusive model.

The second effect of the transport-diffusion coupling is to obtain an error estimate that depends on the norm of a function whose support is spatially restricted to $\Omega_{\mathrm{di}}$. As a consequence, an exact transport solution with large oscillations on $\Omega_{\mathrm{tr}}$ and small oscillations on $\Omega_{\mathrm{di}}$ will imply a smaller norm for $\psi_{2}^{c}$, whose support is restricted to $\Omega_{\mathrm{di}}$, than if the whole problem were treated within the diffusion approximation.

We present these results as follows.

Theorem 3.2. Under the hypotheses of Theorem 3.1 and assuming that the solution $\psi_{2}^{c}$ defined by (31) belongs to $\mathcal{V}\left(\Omega_{\mathrm{di}} \times V\right)$, we have

$$
a_{\varepsilon}^{1 / 2}\left(\psi_{\varepsilon}-\psi_{\varepsilon}^{c}, \psi_{\varepsilon}-\psi_{\varepsilon}^{c}\right) \leq C\left\{\begin{array}{rll}
\varepsilon\left\|\psi_{2}^{c}\right\|_{\mathcal{V}\left(\Omega_{\mathrm{di}} \times V\right)} & \text { if } & \partial \Omega \cap \partial \Omega_{\mathrm{di}} \neq \emptyset \\
\varepsilon^{2}\left\|\psi_{2}^{c}\right\|_{\mathcal{V}\left(\Omega_{\mathrm{di}} \times V\right)} & \text { if } & \partial \Omega \cap \partial \Omega_{\mathrm{di}}=\emptyset
\end{array}\right.
$$

Remark 3.3. In Theorems 3.1 and 3.2 , the coupling interface $\gamma$ is in the domain of validity of the diffusion approximation. This hypothesis is crucial, for the diffusion approximation does not hold in the vicinity of heterogeneities that violate the assumptions of weak absorption and strong scattering $[11,16]$. The coupling interface must be a least a few mean free paths away from the heterogeneities in order for the coupling to be efficient. This will be verified numerically in Section 6 .

\section{ITERATIVE SCHEME FOR THE COUPLED PROBLEM}

A natural method to solve (27) is to iteratively solve the transport problem in $\Omega_{\operatorname{tr}} \times V$ and the diffusion problem in $\Omega_{\mathrm{di}}$, and use the two interface conditions to transmit information across $\gamma$. This method is reminiscent of the relaxation procedure used to solve elliptic and hyperbolic/elliptic problems by domain decomposition in [26] and [19]. 
To simplify, we assume that $\Omega_{\mathrm{tr}}$ is a connected smooth domain and that $\partial \Omega_{\mathrm{tr}} \cap \partial \Omega=\emptyset$; i.e., $\overline{\Omega_{\mathrm{tr}}} \subset \Omega$, and also set $\varepsilon=1$. Following [26] and [19], we introduce the following spaces

$$
\begin{aligned}
V_{\mathrm{di}} & =\left\{\theta \in H^{1}\left(\Omega_{\mathrm{di}}\right), \theta=0 \text { on } \partial \Omega\right\}, \\
V_{\mathrm{di}}^{0} & =H_{0}^{1}\left(\Omega_{\mathrm{di}}\right), \\
V_{\mathrm{tr}} & =\left\{\theta \in \mathcal{V}\left(\Omega_{\mathrm{tr}} \times V\right)\right\}, \\
V_{\mathrm{tr}}^{0} & =\left\{\theta \in \mathcal{V}\left(\Omega_{\mathrm{tr}} \times V\right), \theta=0 \text { on } \gamma_{-}\right\}, \\
\Phi & =\left\{\theta_{\mid \gamma}, \theta \in H^{1}\left(\Omega_{\mathrm{di}}\right)\right\} .
\end{aligned}
$$

We also have that $\Phi=H^{1 / 2}(\gamma)$. This allows us to define the lifting operators $R_{\mathrm{tr}} \phi$ and $R_{\mathrm{di}} \phi$ of $\phi \in \Phi$ in $\Omega_{\mathrm{tr}}$ and $\Omega_{\mathrm{di}}$, respectively, as

$$
\begin{array}{lll}
R_{\mathrm{tr}} \phi \in V_{\mathrm{tr}}: a_{\mathrm{tr}}\left(R_{\mathrm{tr}} \phi, \theta\right)=0 & \forall \theta \in V_{\mathrm{tr}}^{0} \quad \text { and } \quad R_{\mathrm{tr}} \phi=\phi \text { on } \gamma \\
R_{\mathrm{di}} \phi \in V_{\mathrm{di}}: a_{\mathrm{di}}\left(R_{\mathrm{di}} \phi, \theta\right)=0 & \forall \theta \in V_{\mathrm{di}}^{0} \quad \text { and } \quad R_{\mathrm{di}} \phi=\phi \text { on } \gamma
\end{array}
$$

where the boundary-condition-free transport and diffusion bilinear forms are given by

$$
\begin{aligned}
& a_{\mathrm{tr}}(\phi, \theta)=\int_{\Omega_{\mathrm{tr}}} \int_{V}\left(\frac{1}{\Sigma} v \cdot \nabla \phi v \cdot \nabla \theta+\sigma_{a} \phi \theta+\sigma_{s}(\phi-\bar{\phi}) \theta\right) \mathrm{d} x \mathrm{~d} \mu(v), \\
& a_{\mathrm{di}}(\phi, \theta)=\int_{\Omega_{\mathrm{di}}}\left(D \nabla \phi \nabla \theta+\sigma_{a} \phi \theta\right) \mathrm{d} x .
\end{aligned}
$$

We verify that the coupled problem $(27)$ is equivalent to finding $\psi^{c}=\left(\psi_{\mathrm{tr}}^{c}, \psi_{\mathrm{di}}^{c}\right) \in V_{\mathrm{tr}} \times V_{\mathrm{di}}$ such that

$$
\begin{aligned}
& a_{\mathrm{tr}}\left(\psi_{\mathrm{tr}}^{c}, \theta\right)=(q, \theta)_{\mathrm{tr}} \quad \forall \theta \in V_{\mathrm{tr}}^{0}, \quad \psi_{\mathrm{tr}}^{c}=\psi_{\mathrm{di}}^{c} \quad \text { on } \quad \gamma, \\
& a_{\mathrm{di}}\left(\psi_{\mathrm{di}}^{c}, \theta\right)=(q, \theta)_{\mathrm{di}}-a_{\mathrm{tr}}\left(\psi_{\mathrm{tr}}^{c}, R_{\mathrm{tr}} \gamma_{0} \theta\right)+\left(q, R_{\mathrm{tr}} \gamma_{0} \theta\right)_{\mathrm{tr}} \quad \forall \theta \in V_{\mathrm{di}},
\end{aligned}
$$

where $(\cdot, \cdot)_{\mathrm{di}}$ is the usual scalar product on $L^{2}\left(\Omega_{\mathrm{di}}\right),(\cdot, \cdot)_{\mathrm{tr}}$ is the usual scalar product on $L^{2}\left(\Omega_{\mathrm{tr}} \times V\right)$, and $\gamma_{0}$ is the operator that maps functions in $V_{\mathrm{di}}^{0}$ to their trace on $\gamma$. We also denote by $\gamma_{0}$ the operator that maps functions in $\mathcal{V}$ to their trace on $\gamma_{-}$. That this operator is well-posed follows from classical trace theorems [3,13]. Note that the first problem in (39) is a Dirichlet type problem whereas the second one is a Neumann type problem.

Following [26] and [19], we propose to solve the decomposed problem (39) using an iterative method based on successively solving problems on $\Omega_{\mathrm{tr}}$ and $\Omega_{\mathrm{di}}$ with relaxation conditions at the interface $\gamma$. Let the initial guess $\psi^{c, 0}=\left(\psi_{\mathrm{tr}}^{c, 0}, \psi_{\mathrm{di}}^{c, 0}\right)$ be given and solve

$$
\begin{aligned}
& a_{\mathrm{tr}}\left(\psi_{\mathrm{tr}}^{c, k}, \theta\right)=(q, \theta)_{\operatorname{tr}} \quad \forall \theta \in V_{\mathrm{tr}} \quad \gamma_{0}\left(\psi_{\mathrm{tr}}^{c, k}\right)=g^{k-1} \quad \text { on } \quad \gamma_{-} \\
& a_{\mathrm{di}}\left(\psi_{\mathrm{di}}^{c, k}, \theta\right)=(q, \theta)-a_{\mathrm{tr}}\left(\psi_{\mathrm{tr}}^{c, k}, R_{\mathrm{tr}} \gamma_{0} \theta\right)+\left(q, R_{\mathrm{tr}} \gamma_{0} \theta\right)_{\mathrm{tr}} \quad \forall \theta \in V_{\mathrm{di}}
\end{aligned}
$$

where $g^{k} \in \Phi$ is given by

$$
g^{k}=\theta_{k} \gamma_{0}\left(\psi_{\mathrm{di}}^{c, k}\right)+\left(1-\theta_{k}\right) g^{k-1} \quad k \geq 1
$$

Here, $\theta_{k}$ is a relaxation parameter chosen to ensure and optimize the convergence of the iterative scheme. Consider the norms

$$
\begin{aligned}
& \|\theta\|_{\mathrm{tr}}^{2}=a_{\mathrm{tr}}(\theta, \theta) \quad \theta \in V_{\mathrm{tr}} \\
& |\|\phi\||=\left\|R_{\mathrm{tr}} \phi\right\|_{\mathrm{tr}} \quad \phi \in \Phi .
\end{aligned}
$$

The speed of convergence of (40) is then estimated in the following result. 
Theorem 4.1. There exists $\theta^{*} \in(0,1]$ such that the following holds. Let $\theta_{\min }>0$ such that $\theta_{\min } \leq \theta_{k}<\theta^{*}$ for all $k$. Then independent of the initial guess $g^{0} \in \Phi$, the solution $\psi^{c, k}$ of (40) converges to that of (39) as $k \rightarrow \infty$. Furthermore, there exists $\bar{\kappa}<1$ such that

$$
\left|\left\|\gamma_{0}\left(\psi_{\mathrm{tr}}^{c, k}-\psi_{\mathrm{tr}}^{c}\right)\right\|\right| \leq \prod_{l=0}^{k} \kappa\left(\theta_{l}\right)\left|\left\|\gamma_{0}\left(\psi_{\mathrm{tr}}^{c, 1}-\psi_{\mathrm{tr}}^{c}\right)\right\|\right| \quad \forall k \geq 0
$$

where $0<\kappa\left(\theta_{l}\right) \leq \bar{\kappa}$ for every $1 \leq l \leq k$.

Proof. The proof of this theorem goes along the same lines as that of Lemma 3.1 and Theorems 3.1 and 3.2 in [26]. We only emphasize the main difference, related to the norms associated with the transport and diffusion lifting operators. Classical trace results in the physical space show the equivalence between the norm in $H^{1 / 2}(\gamma)$ and $\|\cdot\|_{\mathrm{di}}=\left(a\left(R_{\mathrm{di}} \cdot, R_{\mathrm{di}} \cdot\right)\right)^{1 / 2}$ and in the phase space between $\left|\|\cdot \mid\|\right.$ and the natural norm of $L^{2}\left(\gamma_{-}, d \xi\right)$ (see $[3,13])$. We therefore obtain that

$$
\sigma=\sup _{\phi \in \Phi} \frac{\left\|R_{\mathrm{tr}} \phi\right\|_{\mathrm{tr}}^{2}}{\left\|R_{\mathrm{di}} \phi\right\|_{\mathrm{di}}^{2}} \quad \text { and } \quad \tau=\sup _{\phi \in \Phi} \frac{\left\|R_{\mathrm{di}} \phi\right\|_{\mathrm{di}}^{2}}{\left\|R_{\mathrm{tr}} \phi\right\|_{\mathrm{tr}}^{2}}
$$

satisfy $\sigma<\infty$ and $\tau=\infty$. Following the proof of Theorem 3.1 in [26], we propose

$$
\kappa(\theta)=\theta^{2} \sigma^{2}+(1-\theta)^{2}
$$

so that

$$
\kappa(\theta)<1 \quad \text { if } \quad 0<\theta<\theta^{*}=\min \left(1, \frac{2}{\sigma^{2}+1}\right)
$$

Moreover, the minimal value of $\kappa$ is reached at $\theta_{\mathrm{opt}}=\left(\sigma^{2}+1\right)^{-1}$ and equals

$$
\kappa_{\mathrm{opt}}=\frac{\sigma}{\sqrt{\sigma^{2}+1}}
$$

This concludes our proof.

\section{COUPLing OF TRANSPORT IN FIRST-ORDER FORMULATION AND DIFFUSION}

The even parity form of transport with its variational formulation lends itself naturally to the derivation of diffusion by orthogonal projection. However, most numerical solutions of transport involve the discretization of transport in its first-order formulation (1). Obviously, (1) and (5) are equivalent through (3) and (4) and

$$
u(x, v)=\psi(x, v)+\psi^{-}(x, v)=\psi(x, v)-\frac{1}{\Sigma(x)} v \cdot \nabla \psi(x, v)
$$

Let us assume that the transport model is used on $\Omega_{\mathrm{tr}}$ and the diffusion model on $\Omega_{\mathrm{di}}$. The solution is denoted by $U(x)$ on $\Omega_{\mathrm{di}}$ and by $u(x, v)$ on $\Omega_{\mathrm{tr}}$. Since $v \cdot \nabla \psi(x, v)=-(-v \cdot \nabla \psi(x,-v))$, we deduce that (27) is equivalent 
to the following coupled problem

$$
\begin{aligned}
& -\operatorname{div} D_{\varepsilon} \nabla U+\sigma_{a \varepsilon} U=q_{\varepsilon} \quad \text { in } \quad \Omega_{\mathrm{di}}, \\
& c_{n} U+\varepsilon D_{\varepsilon} \frac{\partial U}{\partial \nu}=0 \quad \text { on } \quad \partial \Omega \cap \partial \Omega_{\mathrm{di}}, \\
& \frac{1}{\varepsilon} v \cdot \nabla u+\sigma_{a \varepsilon} u+\frac{1}{\varepsilon^{2}} \sigma_{s \varepsilon}(u-\bar{u})=q_{\varepsilon} \quad \text { in } \Omega_{\mathrm{tr}} \times V, \\
& u=0 \quad \text { on } \quad \Gamma_{-} \cap\left(\partial \Omega_{\mathrm{tr}} \times V\right), \\
& \frac{1}{2}(u(x, v)+u(x,-v))=U(x) \quad \text { on } \quad \gamma_{-}, \\
& \int_{V} v \cdot \nu_{\gamma}(x) u(x, v) \mathrm{d} \mu(v)=\varepsilon D_{\varepsilon} \frac{\partial U}{\partial \nu_{\gamma}} \text { on } \gamma .
\end{aligned}
$$

The boundary condition involving $u(x, v)$ and $u(x,-v)$ on $\gamma_{-}$is not convenient in practice as most transport algorithms impose Dirichlet boundary conditions on $u(x, v)$ on $\gamma_{-}$. In the diffusive regime, the condition that the even parity transport $\psi(x, v)=U(x)+O\left(\varepsilon^{2}\right)$ can be recast as $u(x, v)=U(x)-\frac{\varepsilon}{\Sigma} v \cdot \nabla U(x)+O\left(\varepsilon^{2}\right)$. Since the interface $\gamma_{-}$is located in a diffusive area, we can replace the coupling condition in (46) on $\gamma_{-}$by

$$
u(x, v)=U(x)-\frac{\varepsilon}{\Sigma} v \cdot \nabla U(x) \quad \text { on } \quad \gamma_{-},
$$

while still retaining an accuracy of order $\varepsilon^{2}$.

The iterative scheme analyzed in the preceding section can then be used to solve the coupled problem (46) with the modification (47) on $\gamma_{-}$. We still assume that $\Omega_{\operatorname{tr}}$ is inside $\Omega$ and set $\varepsilon=1$ to simplify notation. We then obtain the following iterative scheme

$$
\begin{aligned}
& v \cdot \nabla u^{k}+\sigma_{a} u^{k}+\sigma_{s}\left(u^{k}-\bar{u}^{k}\right)=q \quad \text { in } \Omega_{\mathrm{tr}} \times V \text {, } \\
& u^{k}=g^{k-1} \quad \text { on } \quad \gamma_{-} \text {, } \\
& -\operatorname{div} D \nabla U^{k}+\sigma_{a} U^{k}=q \quad \text { in } \quad \Omega_{\mathrm{di}}, \\
& c_{n} U^{k}+D \frac{\partial U^{k}}{\partial \nu}=0 \quad \text { on } \quad \partial \Omega \cap \partial \Omega_{\mathrm{di}}, \\
& \int_{V} v \cdot \nu_{\gamma}(x) u^{k}(x, v) \mathrm{d} \mu(v)=D \frac{\partial U^{k}}{\partial \nu_{\gamma}} \quad \text { on } \quad \gamma,
\end{aligned}
$$

where

$$
g^{k}=\theta_{k}\left(U^{k}-\frac{1}{\Sigma} v \cdot \nabla U^{k}\right)+\left(1-\theta_{k}\right) g^{k-1} \quad k \geq 1
$$

This is the iterative used in the numerical simulations presented in the following section.

\section{Numerical Simulations}

This section presents numerical simulations that emphasize the ability of the coupled transport-diffusion model to capture strong local variations of the particle density. The space dimension is $n=2$. The spatial domain is the unit square $\Omega=(0,1)^{2}$ and the velocity domain the unit circle $V=S^{1}$. The "exact" transport solution, where $\Omega_{\mathrm{tr}}=\Omega$, is compared to the coupled solution of (48) and to the diffusion solution, where $\Omega_{\mathrm{di}}=\Omega$. 


\subsection{Discretizations}

There exist numerous methods for the discretization of the transport equation (1) and the diffusion equation (20). The transport equation is discretized here by using the Diamond Discretization (DD) method. The velocity variable is discretized by $N_{a}$ uniformly distributed points on the unit circle: $(2 i-1) \pi / N_{a}$ for $i=1, \ldots, N_{a}$. The square $\Omega$ is discretized by $N_{x} \times N_{y}$ uniform cells of area $h_{x} \times h_{y}$ where $h_{x}=N_{x}^{-1}$ and $h_{y}=N_{y}^{-1}$. In DD, the discrete solution is constant on each edge of the cells. Averaging (1) over each cell and imposing that the average of the upper and bottom edges be equal to the average of the left and right edges for each cell provides enough equations to uniquely solve the discrete transport equation; we refer to [25] for the details. The diffusion equation is also solved by using a second order accurate discretization based on the $Q_{1}$ finite element method. The discrete diffusion solution and the test functions are continuous and linear both in $x$ and $y$ on every cell of the discretization (see for instance [15]). The codes have been written in Matlab and run on a PC (500 $\mathrm{MHz}$ Pentium III processor) with Linux environment. In all the numerical simulations presented in this paper, the number of discretization points is $N_{x}=N_{y}=160$ and $N_{a}=64$. These figures are necessary to ensure the spatial convergence of the velocity averaged particle density, especially in the presence of weakly scattering regions. The relaxation parameter $\theta$ has been kept fixed and equal to 0.5. We refer to Section 4 and [26] for the details on the optimal choice of $\theta$, which is not considered here.

The number of unknowns in the transport equation, of the order of $N_{a} \times N_{x} \times N_{y}$ is too large to allow for the construction of a matrix and its numerical inversion. This limitation is even more restrictive in three space dimensions, where the number of degrees of freedom is five. The most commonly used method is then an iterative method called source iteration method, which we now present for the continuous equation to simplify. Starting from $u^{0}=0$, we successively solve

$$
\begin{aligned}
& v \cdot \nabla u^{k+1}(x, v)+\Sigma u^{k+1}(x, v)=\sigma_{s} \int_{S^{1}} u^{k}\left(x, v^{\prime}\right) \mathrm{d} \mu\left(v^{\prime}\right)+S(x) \quad \text { in } \Omega \times V, \\
& u^{k+1}=g(x, v) \quad \text { on } \quad \Gamma_{-} .
\end{aligned}
$$

The source iteration method is known to converge very slowly in the diffusive regime, when $\sigma_{s} / \Sigma$ is close to 1 and $\sigma_{s}$ is large compared to the diameter of $\Omega$. The speed of convergence of the method is close to $\sigma_{s} / \Sigma$ and can require thousands of iterations before a satisfactory solution is obtained. The source iteration method does not capture the low frequencies, which are on the other hand very well described by the diffusion approximation. Source iteration methods are therefore almost always preconditioned by a diffusion solver. One major concern about this preconditioning is that the transport and diffusion discretizations must be consistent; see for instance [2]. When consistency is violated, spurious modes may appear for sufficiently large mesh sizes and render the whole iteration method unstable. We refer to the recent review article [1] for more details. Consistent diffusion discretizations however are often quite expensive. We have used here the $Q_{1}$ diffusion discretization as a preconditioner for the Diamond Discretization. Although theoretically not shown to be consistent, the acceleration has proved to be stable when $\Omega=\Omega_{\mathrm{tr}}$ (full transport solution) both in the case of scattering and non-scattering domains. However, the acceleration technique was not stable when solving the coupled problem (48) iteratively. It has therefore been turned off in the calculations of the coupled solution.

\subsection{Extrapolation Length}

The results of Theorem 3.2 show that the solution of the coupled problem (46) is an approximation of the transport solution of order $\varepsilon$ when $\partial \Omega \cap \partial \Omega_{\mathrm{di}} \neq \emptyset$, which is the case we will consider shortly. The reason for this error of order $\varepsilon$ and not $\varepsilon^{2}$ is that the diffusion approximation does not correctly capture the leakage of particles at the domain boundary. Nevertheless, although the transport solution genuinely depends on the velocity variable in the vicinity of the boundary, its average can be accounted for by a diffusion model provided that a careful boundary layer analysis is performed. We refer to $[11,16]$ for details on this analysis. The 

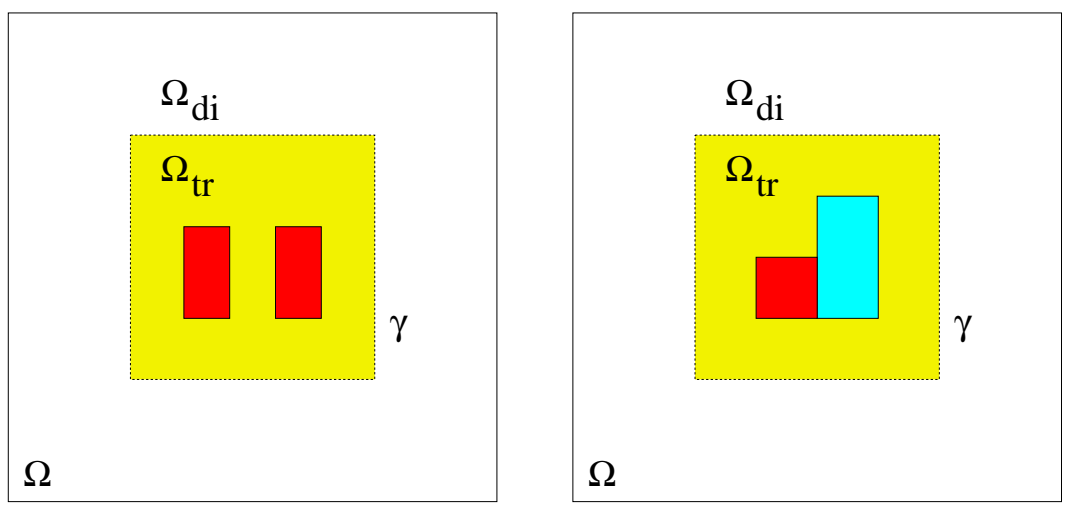

FiguRE 1. Left: geometry of the first setting; Right: geometry of the second setting.

boundary layer appears in the diffusion model in the form of a corrected extrapolation length. Let us denote by

$$
L_{n, d i}=\frac{1}{n c_{n}},
$$

the diffusion extrapolation length in (46). We have $L_{2, d i}=\pi / 4$ and $L_{3, d i}=2 / 3$ in dimensions 2 and 3 , respectively $[9,16]$. The corrected extrapolation lengths obtained from the boundary layer analysis approximately equal

$$
L_{2, t r}=L_{2} \approx 0.8164 \quad \text { and } \quad L_{3, t r}=L_{3} \approx 0.7104
$$

in dimensions 2 and 3, respectively [10,16]. In all numerical simulations below, for which $n=2$, the second equation in (46), and similarly the fourth equation in (48), are replaced by

$$
U+2 L_{2} D \frac{\partial U}{\partial \nu}=0 \quad \text { on } \quad \partial \Omega
$$

\subsection{Physical setting}

The domain $\Omega$ is assumed to be highly scattering, $\sigma_{s}=50$, and weakly absorbing, $\sigma_{a}=0.05$, except in the inclusions.

We consider two settings. In the first setting, two strongly absorbing inclusions are located at $\Omega_{a}=$ $(0.4,0.45)^{2} \cup(0.4,0.45) \times(0.55,0.6)$ and characterized by $\sigma_{a}=20$ and $\sigma_{s}=50$. These coefficients may model the propagation of near infra red photons in a hematoma (whole blood), although the absorption coefficient has been voluntarily magnified to display the error made by using the diffusion approximation. A fictitious interface $\gamma$ surrounds then both inclusions and separates the transport and diffusion domains $\Omega_{\mathrm{tr}}$ and $\Omega_{\mathrm{di}}$; see Figure 1 .

In the second setting, only one inclusion is strongly absorbing and scattering. It is located at $\Omega_{a}=(0.4,0.5)^{2}$ and characterized by $\sigma_{a}=20$ and $\sigma_{s}=50$. A second inclusion is almost non-scattering and non-absorbing (void). It is located at $\Omega_{v}=(0.5,0.6) \times(0.4,0.6)$ and characterized by $\sigma_{a}=\sigma_{s}=0.05$; see Figure 1 . These coefficients may correspond to those of near infra red photons propagating in cerebrospinal fluid within the ventricles at the center of the human head. These hematoma and ventricles are known to hamper the use of the diffusion approximation in diffusion tomography [21].

For both cases, the incoming distribution of particles in (1) is given by the Gaussian beam

$$
g(x, 0, v)=e^{-65(x-0.5)^{2}}
$$




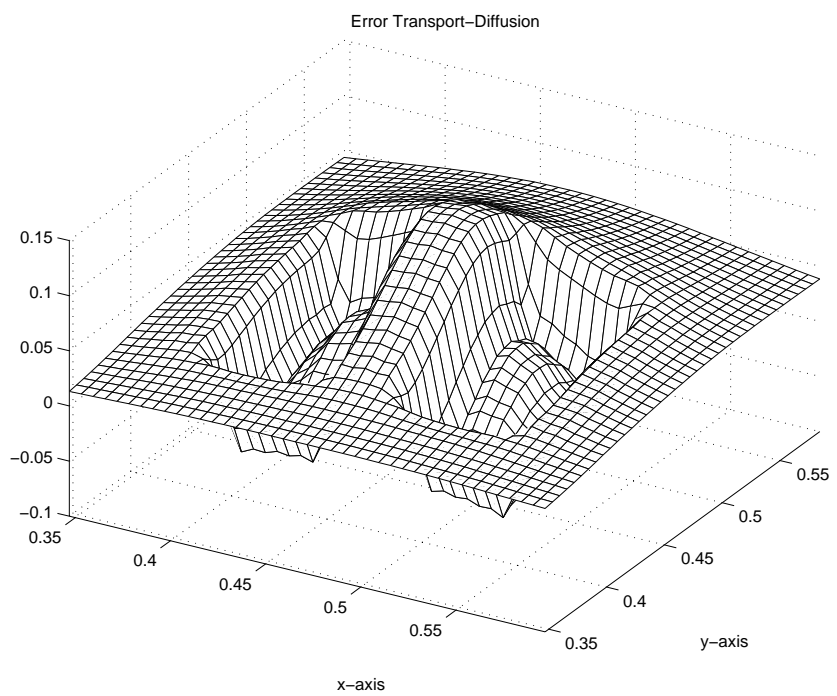

Figure 2. Relative error $e_{\mathrm{d}}(x)$ in the vicinity of the strongly absorbing inclusions. The local variations induced by the inclusions are not accurately resolved by the diffusion model.

on the bottom side. The incoming distribution on the other sides and the volume sources $q$ are set to zero.

We denote by $u(x, v)$ the solution of the transport equation $(1)$, by $u_{\mathrm{c}}(x, v)$ the solution of the coupled problem (46), and by $U(x)$ the solution of the diffusion problem (20) with boundary conditions

$$
U+2 L_{2} D \frac{\partial U}{\partial \nu}=g(x) \quad \text { on } \quad \partial \Omega
$$

We introduce the following relative error terms

$$
e_{\mathrm{c}}(x)=\frac{\int_{V}\left(u(x, v)-u_{\mathrm{c}}(x, v)\right) \mathrm{d} \mu(v)}{\int_{V} u(x, v) \mathrm{d} \mu(v)}, \quad e_{\mathrm{d}}(x)=\frac{\int_{V} u(x, v) \mathrm{d} \mu(v)-U(x)}{\int_{V} u(x, v) \mathrm{d} \mu(v)} .
$$

\subsection{Numerical results}

Let us consider the first setting with two strongly absorbing inclusions. The diffusion model cannot capture the fast oscillations generated by the alternation of highly and weakly absorbing regions. The relative error $e_{\mathrm{d}}$ is displayed in Figure 2 in the vicinity of the inclusions.

Let us first set $\Omega_{\mathrm{tr}}=\Omega_{\mathrm{tr}}^{1}=(0.3,0.7)^{2}$. The absorbing inclusions are well within the domain treated in transport. The numerical results in Figure 3 show that the coupled solution (dashed lines) perfectly captures the local oscillations in the vicinity of the inclusions (right figure). However, the current of particles at the upper side of $\Omega$, given by $D(x) \frac{\partial U}{\partial \nu}(x)$ in the diffusion model and by $\int_{V} v \cdot \nu(x) u(x, v) \mathrm{d} \mu(v)$ in the transport model, is not well approximated by the coupled model, which is about as accurate as the diffusion model. The diffusion model does not capture the microscopic variations generated by the inclusions. However it does capture most of its macroscopic effects. The error in the currents at the upper edge made by the coupled problem is not related to the presence of the inclusion, but rather to the fact that most of the domain is treated within the diffusion approximation. This observation is confirmed by the $L^{2}$-norm of the relative errors $e_{\mathrm{c}}$ and $e_{\mathrm{d}}$. Table 1 shows that $e_{\mathrm{c}}$ is one order of magnitude smaller than $e_{\mathrm{d}}$ in the vicinity of the inclusions. Nevertheless, the global errors on the whole domain $\Omega$ are comparable for the coupled and diffusion models. 

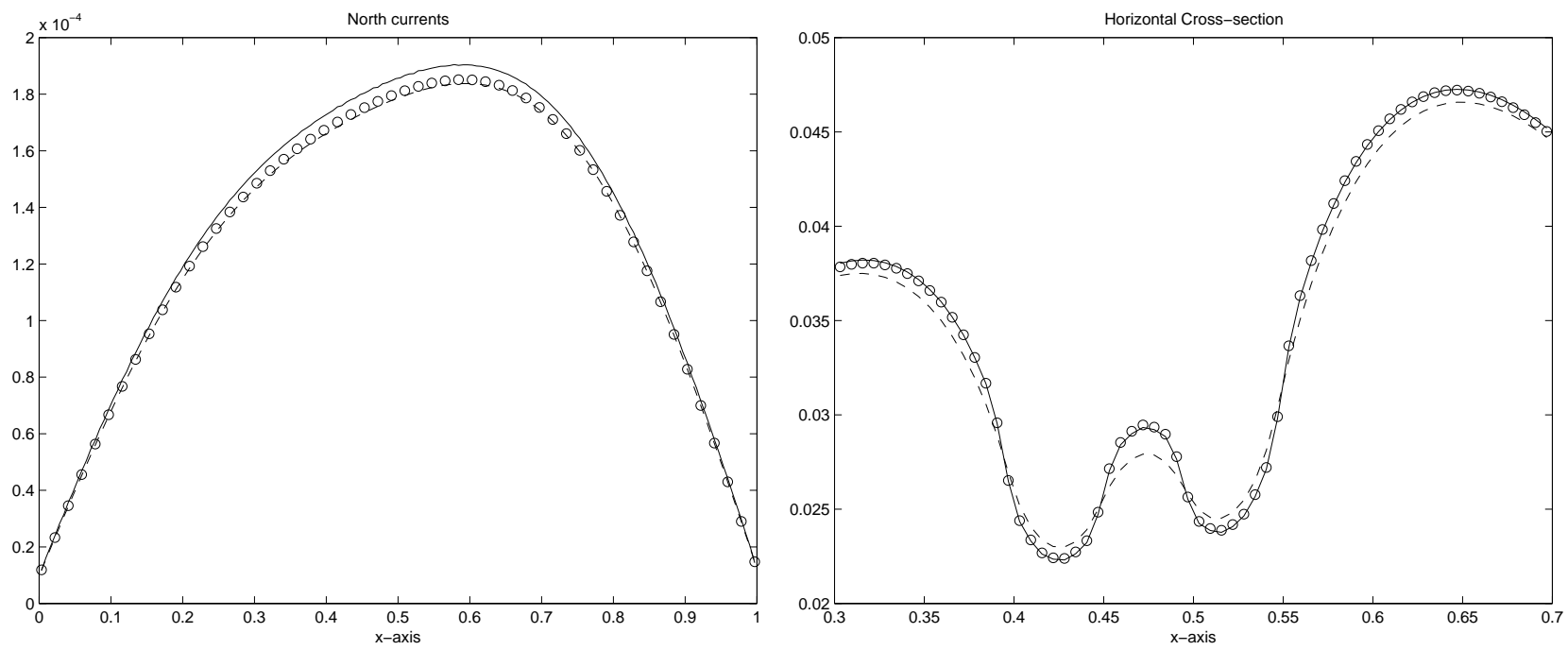

Figure 3. Transport solution (solid lines), coupled solution (circles), and diffusion solution (dashed lines) in the first setting. The domain treated in transport in the coupled problem is $\Omega_{\mathrm{tr}}=(0.3,0.7)^{2}$. The left figure represents the current on the upper side of $\Omega$. The right figure displays the different solutions averaged over the velocity variable on the horizontal cross section $y=0.41$ and $0.3 \leq x \leq 0.7$.

TABLE 1. $L^{2}$-norm of the relative error terms $e_{\mathrm{c}}$ and $e_{\mathrm{d}}$. The transport domain is $\Omega_{\mathrm{tr}}^{1}=(0.3,0.7)^{2}$.

\begin{tabular}{|c|c|c|}
\hline Error & $e_{\mathrm{c}}$ & $e_{\mathrm{d}}$ \\
\hline$|\Omega|^{-1}\|e\|_{L^{2}(\Omega)}$ & $1.7410^{-2}$ & $2.8610^{-2}$ \\
\hline$\left|\Omega_{\mathrm{tr}}^{1}\right|^{-1}\|e\|_{L^{2}\left(\Omega_{\mathrm{tr}}^{1}\right)}$ & $5.2910^{-3}$ & $5.6910^{-2}$ \\
\hline
\end{tabular}

As emphasized in Remark 3.3, the theoretical estimates of Theorem 3.2 hold when the coupling interface is well within the domain of validity of diffusion. Let us now consider the case $\Omega_{\mathrm{tr}}=\Omega_{\mathrm{tr}}^{2}=(0.4,0.55) \times(0.4,0.5)$. Here, the transport model is used only on the absorbing inclusions themselves and the space in between. The results of Table 1 are replaced by those of Table 2 . Notice that the norm of $e_{\mathrm{c}}$ on $\Omega_{\mathrm{tr}}$ and its norm on $\Omega_{\mathrm{di}}$ are now comparable. There is still a gain in solving the heterogeneities in transport, but the improvement is less clear than when the boundary layer terms are also fully resolved. Practical calculations suggest that a layer of at least 2-5 mean free paths around the heterogeneities need be solved by transport to account for the boundary layer.

TABLE 2. $L^{2}$-norm of the relative error terms $e_{\mathrm{c}}$ and $e_{\mathrm{d}}$. The transport domain is $\Omega_{\mathrm{tr}}^{2}=(0.4,0.55) \times(0.4,0.5)$.

\begin{tabular}{|c|c|c|}
\hline Error & $e_{\mathrm{c}}$ & $e_{\mathrm{d}}$ \\
\hline$|\Omega|^{-1}\|e\|_{L^{2}(\Omega)}$ & $1.8810^{-2}$ & $2.8510^{-2}$ \\
\hline$\left|\Omega_{\mathrm{tr}}^{2}\right|^{-1}\|e\|_{L^{2}\left(\Omega_{\mathrm{tr}}^{2}\right)}$ & $2.0510^{-2}$ & $5.7010^{-2}$ \\
\hline
\end{tabular}

The same calculations are now performed in the second setting. Unlike the case of absorbing inclusions, the diffusion model performs very poorly in the presence of weakly scattering inclusions. Weak scattering is 


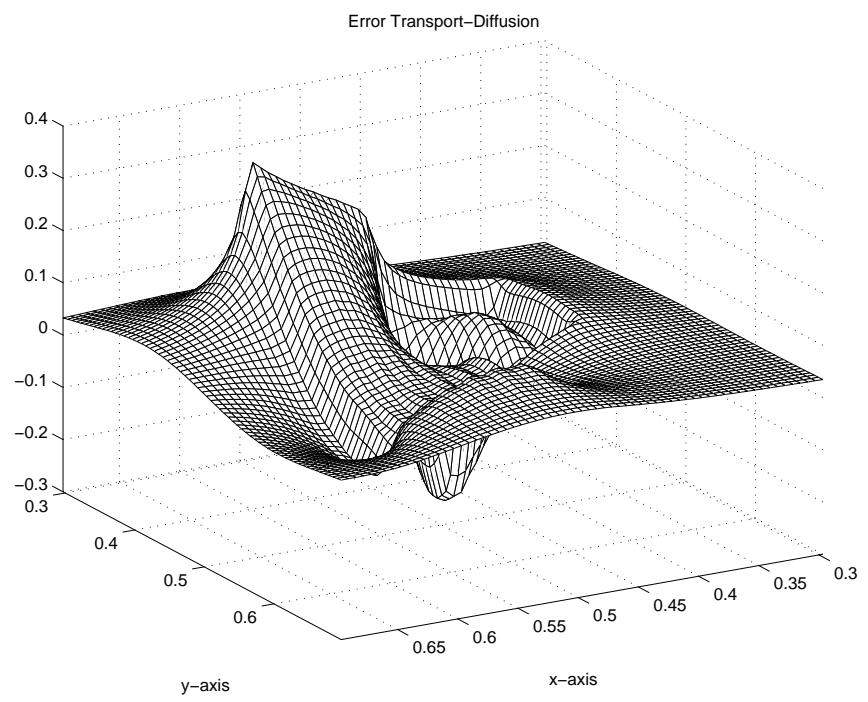

Figure 4. Relative error $e_{\mathrm{d}}(x)$ in the vicinity of the inclusions in the second setting. The domain $\Omega$ is seen from behind.

tantamount to large diffusion coefficients, which have the effect of amplifying the dispersion of particles within the inclusion; see for instance $[4,8,18,21]$. In addition, diffusion still cannot capture fast oscillations of the particle density. The relative error $e_{\mathrm{d}}$ is displayed in Figure 4 in the vicinity of the inclusions. In addition to capturing local heterogeneities very poorly, the diffusion model underestimates the density of particles in the lower part of the weakly absorbing inclusion and overestimates it in its upper part. The reason is that by overestimating the dispersiveness of the inclusion, the diffusion model causes more particles to travel upwards than is physically correct.

We set $\Omega_{\mathrm{tr}}=(0.3,0.7)^{2}$. Again, both inclusions are about 5 mean free path within the transport domain $\Omega_{\mathrm{tr}}$ so that the boundary layers are correctly resolved. Unlike the previous case, the weakly scattering inclusion is so poorly treated by the diffusion model that the impact can be seen in the whole domain $\Omega$. The upper part of Figure 5 shows that, albeit not perfect, the current calculated with the coupled model has a much better fit to the transport current than the one with diffusion, even though most of the area of the coupled problem is still solved by diffusion. Notice in the right part of Figure 5 that local variations are perfectly reproduced by the coupled problem. In zones of large diffusion coefficient, the particle density is almost flat as particles entering this area are instantly diffused away. This is well displayed by the flat part of the diffusion calculation (dashed lines, $0.5<x<0.6)$.

All these observations are also confirmed by the $L^{2}$-norm of the relative errors $e_{\mathrm{c}}$ and $e_{\mathrm{d}}$. Table 3 shows that $e_{\mathrm{c}}$ is not only two orders of magnitude smaller than $e_{\mathrm{d}}$ in the vicinity of the inclusions but also significantly smaller in the whole domain.

TABLE 3. $L^{2}$-norm of the relative error terms $e_{\mathrm{c}}$ and $e_{\mathrm{d}}$. The transport domain is $\Omega_{\mathrm{tr}}^{1}=(0.3,0.7)^{2}$.

\begin{tabular}{|c|c|c|}
\hline Error & $e_{\mathrm{c}}$ & $e_{\mathrm{d}}$ \\
\hline$|\Omega|^{-1}\|e\|_{L^{2}(\Omega)}$ & $9.7010^{-3}$ & $4.5210^{-2}$ \\
\hline$\left|\Omega_{\mathrm{tr}}\right|^{-1}\|e\|_{L^{2}\left(\Omega_{\mathrm{tr}}^{1}\right)}$ & $2.6910^{-3}$ & $1.5010^{-1}$ \\
\hline
\end{tabular}



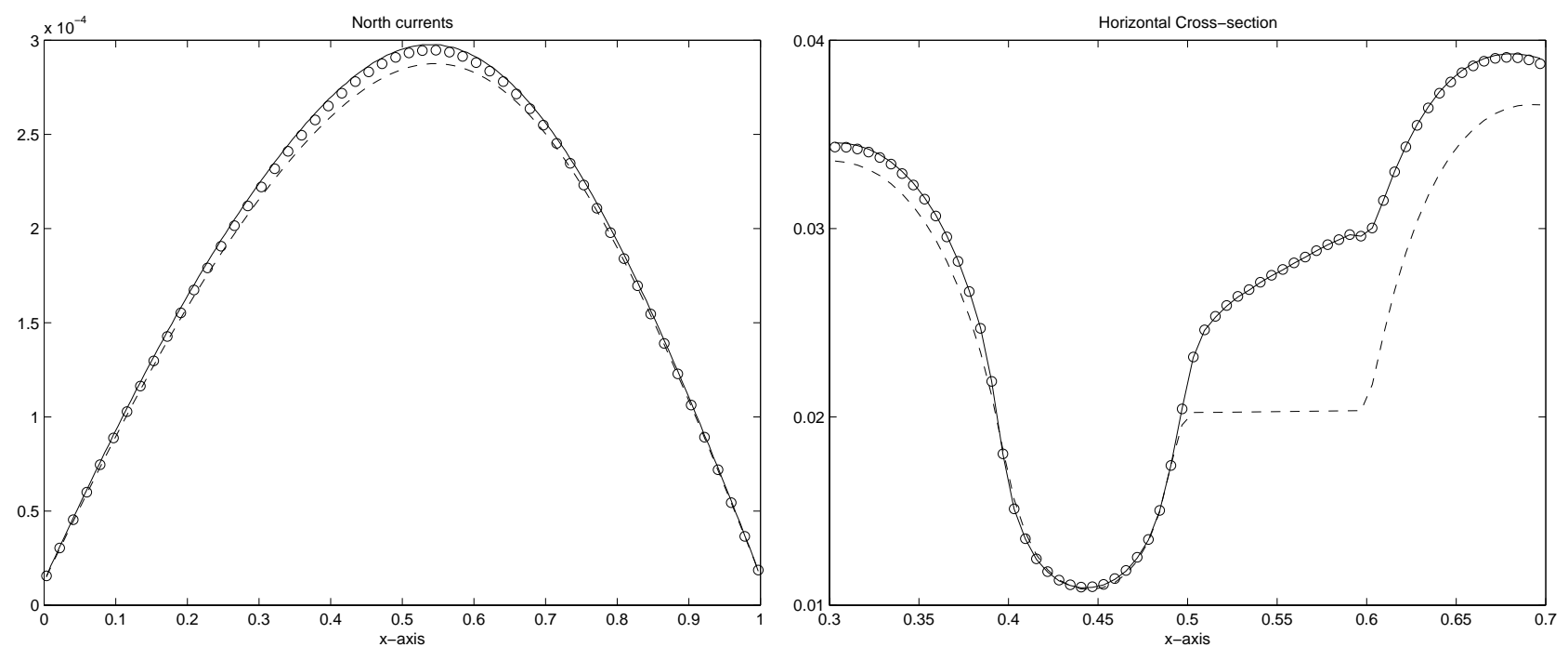

Figure 5. Transport solution (solid lines), coupled solution (circles), and diffusion solution (dashed lines) in the second setting. The domain treated in transport in the coupled problem is $\Omega_{\mathrm{tr}}=(0.3,0.7)^{2}$. The left figure represents the current on the upper side of $\Omega$. The right figure displays the different solutions on the horizontal cross section $y=0.41$ and $0.3 \leq x \leq 0.7$.

Acknowledgements. The authors would like to acknowledge very stimulating discussions during the events organized by the French G.D.R. Couplage d'Équations. The first author was supported in part by NSF Grant DMS-0072008.

\section{REFERENCES}

[1] M.L. Adams and E.W. Larsen, Fast iterative methods for deterministic particle transport computations. Preprint (2001).

[2] R.E. Alcouffe, Diffusion synthetic acceleration methods for the diamond-differenced discrete-ordinates equations. Nucl. Sci. Eng. 64 (1977) 344.

[3] G. Allaire and G. Bal, Homogenization of the criticality spectral equation in neutron transport. ESAIM: M2AN 33 (1999) $721-746$.

[4] S.R. Arridge, H. Dehghani, M. Schweiger, and E. Okada, The finite element model for the propagation of light in scattering media: A direct method for domains with nonscattering regions. Med. Phys. 27 (2000) 252-264.

[5] G. Bal, Couplage d'équations et homogénéisation en transport neutronique. Thèse de Doctorat de l'Université Paris 6 (1997). In French.

[6] G. Bal, First-order corrector for the homogenization of the criticality eigenvalue problem in the even parity formulation of the neutron transport. SIAM J. Math. Anal. 30 (1999) 1208-1240.

[7] G. Bal,Spatially varying discrete ordinates methods in $X Y$-geometry. $M^{3} A S$ (Math. Models Methods Appl. Sci.) 10 (2000) $1277-1303$.

[8] G. Bal, Transport through diffusive and non-diffusive regions, embedded objects, and clear layers. To appear in SIAM J. Appl. Math.

[9] G. Bal, V. Freilikher, G. Papanicolaou, and L. Ryzhik, Wave transport along surfaces with random impedance. Phys. Rev. B 6 (2000) 6228-6240.

[10] G. Bal and L. Ryzhik, Diffusion approximation of radiative transfer problems with interfaces. SIAM J. Appl. Math. 60 (2000) $1887-1912$

[11] A. Bensoussan, J.-L. Lions, and G. Papanicolaou, Boundary layers and homogenization of transport processes. Res. Inst. Math. Sci. Kyoto Univ. 15 (1979)53-157.

[12] J.-F. Bourgat, P. Le Tallec, B. Perthame, and Y. Qiu, Coupling Boltzmann and Euler equations without overlapping, in Domain Decomposition Methods in Science and Engineering, The Sixth International Conference on Domain Decomposition, Como, Italy, June 15-19, 1992, Contemp. Math. 157, American Mathematical Society, Providence, RI (1994) 377-398.

[13] M. Cessenat, Théorèmes de trace $L^{p}$ pour des espaces de fonctions de la neutronique. C. R. Acad. Sci. Paris Sér. I Math. 299 (1984) 831-834. 
[14] S. Chandrasekhar, Radiative Transfer. Dover Publications, New York (1960).

[15] P.G. Ciarlet, The Finite Element Method for Elliptic Problems. North-Holland Publishing Company, Amsterdam, New York, Oxford (1978).

[16] R. Dautray and J.-L. Lions, Mathematical Analysis and Numerical Methods for Science and Technology 6. Springer-Verlag, Berlin (1993).

[17] J.J. Duderstadt and W.R. Martin, Transport Theory. Wiley-Interscience, New York (1979).

[18] M. Firbank, S.A. Arridge, M. Schweiger, and D.T. Delpy, An investigation of light transport through scattering bodies with non-scattering regions. Phys. Med. Biol. 41 (1996) 767-783.

[19] F. Gastaldi, A. Quarteroni, and G. Sacchi Landriani, On the coupling of two-dimensional hyperbolic and elliptic equations: analytical and numerical approach, in Domain Decomposition Methods for Partial Differential Equations, Third International Symposium on Domain Decomposition Methods for Partial Differential Equations, Houston, TX, 1989, SIAM, Philadelphia, PA (1990) 22-63.

[20] F. Golse, P.-L. Lions, B. Perthame, and R. Sentis, Regularity of the moments of the solution of a transport equation. J. Funct. Anal. 76 (1988) 110-125.

[21] A.H. Hielscher, R.E. Alcouffe, and R.L. Barbour, Comparison of finite-difference transport and diffusion calculations for photon migration in homogeneous and heterogeneous tissues. Phys. Med. Biol. 43 (1998) 1285-1302.

[22] A. Ishimaru, Wave Propagation and Scattering in Random Media. Academics, New York (1978).

[23] B. Lapeyre, E. Pardoux and R. Sentis, Méthodes de Monte-Carlo pour les équations de transport et de diffusion, in Mathématiques \& Applications 29, Springer-Verlag, Berlin (1998).

[24] C.D. Levermore, W.J. Morokoff and B.T. Nadiga, Moment realizability and the validity of the Navier-Stokes equations for rarefied gas dynamics. Phys. Fluids 10 (1998) 3214-3226.

[25] E.E. Lewis and W.F. Miller Jr., Computational Methods of Neutron Transport. John Wiley \& Sons, New York (1984).

[26] L.D. Marini and A. Quarteroni, A Relaxation procedure for domain decomposition methods using finite elements. Numer. Math. 55 (1989) 575-598.

[27] J. Planchard, Méthodes mathématiques en neutronique, in Collection de la Direction des Études et Recherches d'EDF, Eyrolles (1995). In French.

[28] L.Ryzhik, G.Papanicolaou, and J.B. Keller, Transport equations for elastic and other waves in random media. Wave Motion 24 (1996) 327-370.

[29] H. Sato and M.C. Fehler, Seismic Wave Propagation and Scattering in the Heterogeneous Earth, in AIP Series in Modern Acoustics and Signal Processing, AIP Press, Springer, New York (1998).

[30] M. Tidriri, Asymptotic analysis of a coupled system of kinetic equations. C. R. Acad. Sci. Paris Sér. I 328 (1999) $637-642$.

[31] S. Tiwari, Application of moment realizability criteria for the coupling of the Boltzmann and Euler equations. Transport Theory Statist. Phys. 29 (2000) 759-783.

To access this journal online:

www.edpsciences.org 